\title{
Alternative medicines worth researching? Citation analyses of acupuncture, chiropractic, homeopathy, and osteopathy 1996-2017
}

\author{
Mike Thelwall ${ }^{1}$ (D)
}

Received: 7 May 2021 / Accepted: 20 August 2021 / Published online: 3 September 2021

(c) Akadémiai Kiadó, Budapest, Hungary 2021

\begin{abstract}
Some complementary and alternative medicines (CAM) are frequently criticised for being based on faith rather than scientific evidence. Despite this, researchers, academic departments, and institutes teach and investigate them. This article assesses whether the scholarship produced by four CAMs is valued by the academic community in terms of citations, and whether the level of citations received might be detrimental to academic authors' careers. Based on an analysis of acupuncture, chiropractic, homeopathy, and osteopathy journal articles indexed in Scopus 1996-2020, the results show that the prevalence of the four areas vary substantially internationally, with acupuncture eclipsing the others in East Asia but homeopathy being more common in India and Brazil. The main broad fields publishing these specialties are Medicine, Nursing, Health Professions, Veterinary Science, and Neuroscience. Whilst the research tends to be cited at a below average rate in most broad fields $(n=27)$ and years (1996-2017), acupuncture, chiropractic, and homeopathy are exceptions in some broad fields, including some core areas. Thus, studying these alternative medicines may not always lead to research that tends to be ignored in academia, even if many scientists disparage it. As a corollary, citation analysis cannot be relied on to give low scores to widely disparaged areas of scholarship.
\end{abstract}

Keywords Alternative medicine $\cdot$ Complementary medicine Acupuncture $\cdot$ Chiropractic . Homeopathy $\cdot$ Osteopathy $\cdot$ Citation analysis $\cdot$ Publication trends $\cdot$ Scopus

\section{Introduction}

Complementary and alternative medicine (CAM) has been defined by the Cochrane Collaboration to be, "a broad domain of resources that encompasses health systems, modalities, and practices and their accompanying theories and beliefs, other than those intrinsic to the dominant health system of a particular society or culture in a given historical period. CAM includes such resources perceived by their users as associated with positive health outcomes.

Mike Thelwall

m.thelwall@wlv.ac.uk

1 Statistical Cybermetrics Research Group, University of Wolverhampton, Wolverhampton, UK 
Boundaries within CAM and between the CAM domain and the domain of the dominant system are not always sharp or fixed" (Zollman \& Vickers, 1999). This definition means that the set of CAM treatments varies between countries (e.g., for Europe: Falkenberg et al., 2012). A treatment might be called alternative if it is proposed as a substitute for modern medicine or complementary if it is for use in conjunction with it. In practice, the terms seem to be used interchangeably for the same treatments and they are used as synonyms here.

Some CAMs are based on unsubstantiated hypotheses about how they might be helpful. For example, a homeopath might believe that water remembers chemicals that had once been dissolved in it, even after the chemicals had been diluted away, and that this memory might trigger the body to fight diseases. There is a large international market for CAM therapies (Ernst, 2000), with at least 170 countries using some form, although the varieties used varies substantially (WHO, 2019). Many countries have government departments to regulate aspects of their preparation or use (e.g., India's Ministry of Ayush) and the World Health Organisation (WHO) has encouraged the integration of traditional medicines into primary care, for example within the Declaration of Astana. Integrative medicine approaches (e.g., in the USA) may incorporate mainstream medicine and CAMs within holistic health plans.

CAMs are taught and researched in some universities to support practitioners. Although without empirical evidence of effectiveness in some cases, students may learn beliefs about suitable remedies for different conditions as well as safety considerations and ethical principles. For research, academics might be interested in testing whether treatments work, investigating the safety of products or use practices, discovering related belief systems in populations, and assessing whether beliefs in alternative medicines undermine willingness to accept modern medicine. Although increasingly many CAM academic studies are being published (Bougioukas et al., 2021; Danell et al., 2020; Treister-Goltzman \& Peleg, 2015), and particularly in Mainland China (Ding \& Li, 2020) and in the USA for some topics (e.g., cancer) (Yang et al., 2019), scholars with a CAM career focus or occasionally engaging with it may worry that their work will not be valued. Similarly, academic institutions may be concerned that some or all CAM research will be disparaged by the wider academic community and will undermine their reputations. This study investigates these issues from a citation analysis perspective for four therapies, assessing whether CAM research meets academic norms in terms of the citations that it attracts. Whilst citations are a very crude indicator of scholarly influence, their availability allows large scale international studies that would not be practical for reputational surveys of researchers.

This article focuses on the prevalence and citation impact of journal articles mentioning four CAMs: acupuncture, chiropractic, homeopathy, and osteopathy. These areas were chosen for pragmatic reasons: they are common and relatively easy to identify in academic publications (see Methods or more details). The purpose of the study is not to shed light on their scientific credentials but to identify where (country and discipline) alternative medicines are researched and whether, from a citation impact perspective, alternative medicines can be valued within academia. All CAM therapies are different and those not investigated should not be assumed to follow similar patterns.

\section{Background}

This section gives a brief introduction to citation analysis and the four therapies investigated. The purpose is not to evaluate these CAMs but to report scientific evidence about them to give context to how they may be perceived in academia. Two common scientific 
criticisms of some alternative medicines are that they may appear to work from a placebo effect rather than their claimed mechanism, and that practitioners and patients may be convinced of their efficacy due to "regression to the mean": if a patient takes any treatment during an illness that they recover from, then they may be convinced that the treatment cured them even if they would have recovered anyway.

\section{Citation analysis}

Citation-based indicators are widely used to support formal and informal research evaluations as well as to map science and identify research fronts (Moed, 2006). The core idea is that a citation tends to indicate that the cited work has helped the citing work, so the citation is to some extent crediting the earlier publication for its contribution (Merton, 1973). Whilst some citations are negative, criticizing or discrediting the earlier paper, and others are perfunctory, such as by setting the research context, on a sufficiently large scale these factors may tend to average out, improving the reliability of the scores. Nevertheless, citations are irrelevant in non-hierarchical areas of science, such as the arts, humanities and some social sciences, and citation counts have systematic national and other biases derived from the incomplete databases used to calculate them (Martín-Martín et al., 2021). There are also human biases from friendship networks and language barriers (Silvello, 2018). Moreover, citations do not reflect the end uses of research, such as commercial products, medical treatments, or policy guidance, so they are only partial indicators of overall value.

Many bibliometric analyses of individual CAMs have used citation analysis, but typically to identify hot topics or connections between research fields rather than to evaluate the impact of an entire field. In one partial exception, a study of all CAMs found that most CAM studies are published in journals specialising in CAMs, and these journals had approximately average citation impact for their fields (Danell et al., 2020). Another study found that CAM papers from East Asia tended to be the most cited (Fu et al., 2011).

\section{Acupuncture}

Acupuncture is a traditional Chinese medicine involving an acupuncturist inserting fine needles into the patient's body. Originally, the position and number of the needles were believed to influence the way that the body was traditionally thought to work, such by affecting its qi or life energy (Anonymous, 1979). Although its popularity declined in China from the 13th Century, it was revitalised in the second half of the twentieth century in China and spread to the West. Western versions often seem to omit the belief-based components and focus on potential biomedical explanations, such as that needle insertion may trigger chemical reactions in the body (White, 2009).

Acupuncture is often claimed to alleviate pain (Linde et al., 2016a, b) amongst other conditions. It can theoretically lead to the release of pain-relieving chemicals in the body, although most studies show that when it works, it is primarily or exclusively a placebo effect. It has been argued that the thousands of acupuncture efficacy trials so far have produced no convincing evidence that it has anything other than a minor effect in any context, so it is illogical to continue to assess it for clinical efficacy (Colquhoun \& Novella, 2013). Nevertheless, it is recommended in the UK as a possible treatment for some types of headache (NHS, 2021), based on two systematic reviews (Linde et al., 2016a, b).

From a bibliometric perspective, different applications of acupuncture have previously been investigated, with Mainland China and the USA being the main producers, and both 
CAM journals and specialist medical journals being major venues. Magnetic Resonance Imaging studies of acupuncture are primarily published by Mainland China and in the journal Evidence-based Complementary and Alternative Medicine (Zhang et al., 2020). The same is true for pain management (Gao et al., 2021) and knee osteoarthritis (Li et al., 2020). Insomnia research is also published most by Mainland China, but in the journal Sleep (Pei et al., 2019). Lower back pain acupuncture research is mostly from the USA and in the Journal of Alternative and Complementary Medicine (Liang et al., 2017). Migrainerelated acupuncture research is most published in the USA and in the headache journal Cephalalgia (Zhao, 2021). The USA also published the most acupuncture research in obstetrics and gynaecology (Wu et al., 2021).

\section{Chiropractic}

Chiropractic mainly involves manual manipulation of the spine, joints, and soft tissues to treat musculoskeletal system disorders. Its US inventor believed that chiropractic procedures may also affect general health. In 2017, 90 countries had at least one chiropractor and 19 provided chiropractic education. Whilst most of these countries had few chiropractors (median 10 for the 90 countries with at least one), there were 77,000 in the USA, supported by 18 educational institutions (Stochkendahl et al., 2019). Chiropractors are by far the most prevalent in the USA, but are also moderately common in Australia, Canada, and the UK.

There is evidence that chiropractic medicine may help back pain to some degree (Walker et al., 2010). Other claims for the key chiropractic spinal manipulation method are not well supported by high quality scientific evidence (Ernst, 2008). There do not seem to have been any chiropractic bibliometrics papers, other those focusing on article types (e.g., Pohlman et al., 2009).

\section{Homeopathy}

Homeopathy is a system of alternative or complementary medicine usually based on the idea that highly diluted solutions of substances that cause symptoms can also treat them, by triggering appropriate bodily responses to them. It originated from Germany in 1796 and has since spread internationally (Relton et al., 2017).

There is a large international homeopathic industry, homeopathy-focused researchers, homeopathy academic departments (including the Faculty of Homeopathy in the UK), qualifications, and some national governmental support. Governmental support in some countries has been criticized as an attempt to pretend to be delivering health care in a situation where modern medicine is not available or affordable, for example as a Covid-19 cure (https://www.sciencemag.org/news/2020/10/fraud-nation-critics-blast-indian-governments-promotion-traditional-medicine-covid-19).

Homeopathy is a controversial subject that is frequently dismissed as a pseudoscience. For example, an Australian review of the effectiveness of homeopathic medicines found that, "there are no health conditions for which there is reliable evidence that homeopathy is effective" (NHMRC, 2015, p. 6). Similarly, the UK National Health Service (NHS) in 2017 after reviewing evidence recommended that homeopathic prescriptions not be funded on the ground of ineffectiveness (BBC, 2018; Davis \& Campbell, 2017). This followed repeated criticism from doctors and falling prescription rates.

Homeopath adherents, including some with scientific credentials, disagree with its criticisms or at least are open to the possibility that it works. Some systematic reviews have 
claimed evidence of an effect of homeopathic remedies but these conclusions are themselves controversial. For example, one study with a tentative positive finding was complicated by the inclusion of remedies that are not highly diluted but nevertheless qualify as homeopathic according to some practitioner definitions (see 26 Feb. 2010 comment on: Kassab, et al., 2009), or that are arguably not homeopathic (see 8 Jan. 2013 comment on an earlier version of: Mathie et al., 2015). This leaves the possibility open that some homeopathic remedies work due to traditionally active ingredients in non-trivial amounts $(20 \%$ in the above case), rather than due to homeopathic principles.

Academic homeopathy research is published mainly by the UK, USA, Germany and India, with the main journal being Homeopathy (Şenel, 2019). In contrast, Germany publishes the most physicochemical research about homeopathic preparations (Klein et al., 2018).

\section{Osteopathy}

Osteopathy mainly involves manipulation of the muscles and bones. It originated in the USA with a belief that manipulation of one part of the body might improve the health of other parts. It is somewhat like chiropractic, but without a focus on the spine and with a different underlying set of beliefs. Elements of osteopathy are recognised by mainstream medicine, and it seems to be effective for lower back pain (Franke et al., 2014), although it is not clear whether it is cost-effective compared to alternatives (Steel, et al., 2017). No bibliometric studies focusing on osteopathy seem to have been published so far.

\section{Methods}

The research design was to calculate the field and year normalized citation score of four CAM fields over a long period to assess whether they tended to be less cited than average. Acupuncture (113 countries), chiropractic (96 countries), osteopathy (94 countries), and homeopathy (100 countries) are amongst the ten alternative medicines most used by countries, according to a World Health Organisation (WHO) survey of 133 member states (WHO, 2019). The other six alternative medicines cover a wide range of practices that could be referred to by many names for their constituent parts (herbal medicines, indigenous traditional medicine, traditional Chinese medicine, naturopathy, Ayurvedic, Unani). Field and year normalization for the citation counts, or a variant such as calculating withinfield, within year citation percentiles, is standard practice in citation analysis when articles from different fields or years need to be compared. This is because citations take time to accrue and arrive at different rates between fields.

\section{Data}

Scopus was chosen as the citation database for the study because it has wider coverage than the Web of Science, which is important because alternative medicines are widely used internationally (WHO, 2019). Moreover, Scopus has better coverage of non-English sources (Mongeon \& Paul-Hus, 2016) and non-English articles (Vera-Baceta et al., 2019), often with English translated titles and abstracts. Dimensions.ai (Thelwall, 2018), Microsoft Academic, and Google Scholar (Martín-Martín, et al., 2021) probably have wider coverage, but Google Scholar lacks a public field classification system and Microsoft 
Academic and Dimensions' classification systems are untested. The years 1996 to 2020 were chosen for analysis. Scopus' coverage expanded greatly in 1996 so earlier data is not comparable. 2020 was the last complete year at the time of data collection (2021). All journal articles in Scopus were downloaded in January-February 2019 (1996-2013) or January 2021 (2014-2020). This gives at least three full years of citation data for every year 1996-2017, which is adequate for most fields (Wang, 2013). Thus, the years 1996-2017 were used for the citation analysis component, but 1996-2020 was used for publishing trends. Sample sizes are in the online supplement (https://doi.org/10.6084/m9.figshare. 14547213).

Acupuncture articles were extracted from the downloaded Scopus set with the query *acupunct*. This matches the text string acupunct within any words (e.g., acupuncture, acupuncturist, electroacupuncture). Similarly, chiropractic articles were extracted with the query * chiropract*, homeopathy with *homeopath*, and osteopathy with *osteopath*. All queries were matched only against the title, abstract or keywords fields. Articles with fewer than 500 characters in their abstracts were excluded, because some articles had short or empty abstracts, especially for older data, so the matching process would be more inclusive for more recent articles, on average, without this restriction.

Articles were assigned to countries based on the national affiliation of the first author, as and when recorded in Scopus. Researchers with multiple affiliations were analysed based on their first affiliation since this seems to be usually a main affiliation, with others being honorary or secondary posts. Although all authors usually contribute to a study, the first author is typically the major contributor except in alphabetically ordered fields, such as some areas of maths and economics (Larivière et al., 2016; Mongeon et al., 2017). Last authors can also be important in some biomedical fields, but this was not taken into account due to a lack of relevant data in Scopus. This should have little effect on the results because it would only alter the assignment for internationally collaborative research in relevant fields with the first and last authors from different countries.

\section{Citation indicators}

Scopus classifies journal articles into 27 broad fields and 325 narrow fields (Scopus, 2021). The broad fields with the most articles were used for the primary analysis and the results for the other broad fields are reported in the online supplement (https://doi.org/10.6084/ $\mathrm{m} 9$.figshare.14547213). The narrow fields were used for the field normalisation process, as described below. The Scopus article classification scheme is mainly based on manual classification of each article's publishing journal, except for multidisciplinary articles which seem to be individually assigned to classifications by Scopus using an algorithm.

The field and year normalized citation count for each article was calculated using the Mean Normalised Log-transformed Citation Score (MNLCS) as described below (Thelwall, 2017). Sets of citation counts are typically highly skewed, with most articles having 0 or few citations and a few articles having many. The arithmetic mean is a poor estimator of central tendency for such a collection because its value is dominated by a small number of highly cited articles. Either the geometric mean or a log transformation can be used to cope with this situation and a log transformation is used within the MNLCS to fit better within a field and year normalisation calculation.

Each article's citation count $c_{0}$ was first log-transformed to reduce skewing, giving $\ln \left(1+c_{0}\right)$. One is added within the formula because some articles are uncited. The average log-transformed citation count for each narrow field and year $\overline{\ln (1+c)}$ was then 
calculated. Each article's citation count was then replaced with a field and year normalized $\log$-transformed citation score (NLCS): $\ln \left(1+c_{0}\right) / \overline{\ln (1+c)}$. For an article in more than one field, the average of the $\ln \left(1+c_{0}\right) / \overline{\ln (1+c)}$ for each field was used instead. For each article, this gives a score that is 1 if the article is as cited as average for its field and year. Scores above 1 equate to more cited than average for the field and year and scores below 1 equate to less cited than the world average. Because of the reduction in skewing, approximately half of the articles have an MNLCS above 1. The MNLCS for acupuncture, chiropractic, homeopathy or osteopathy in any given field and year (or overall for a year) is then the average NLCS for all qualifying articles. By design, it is reasonable to compare these figures between fields and years.

Although the MNLCS for all articles in any given field is close to 1 by design, it is not exactly 1 because of the averaging of the values between fields for articles classified within multiple fields. To cope with this, MNLCS values for any given broad field and year were corrected by adding or subtracting the amount necessary to reach 1, with the same applied to any subset (e.g., the acupuncture articles).

\section{Results}

\section{International and disciplinary prevalence of research about alternative medicine}

For the 30 countries with the most journal articles indexed in Scopus, acupuncture seems to be the most researched of the four methods in most cases (Fig. 1), although this may be due to other methods being more frequently mentioned indirectly in titles, keywords, and abstracts. The results may also ignore national literature not indexed by Scopus that may contain the vast majority of literature of some alternative medicines in some countries.

Acupuncture seems to be by far the most common of the four approaches in East Asia, judging by the results for Mainland China, Taiwan, Hong Kong, Japan, and South Korea, with the remaining alternative medicines being largely ignored. In contrast, chiropractic is the most common of the four alternative medicines in Canada and Denmark. Homeopathy is most common overall for India and is also relatively common in Brazil, Switzerland, Germany (its home country), Israel, Austria, the UK, and Norway. Osteopathy is not common anywhere but is the most common of the four methods in the Czech Republic. Overall, there are large international differences in the uptake of the methods, with none being universally accepted or rejected. The countries with apparently the least research interest in any method are the Czech Republic, Finland, the Russian Federation, and Poland.

Unsurprisingly, journals from health and medicine-related fields publish most papers about the four alternative medicines (Fig. 2). The apparent discrepancy between Fig. 1 (dominated by acupuncture) and Fig. 2 (more balanced) is mainly due to Medicine being a huge field. It is perhaps interesting that there is substantial veterinary research interest in all four alternative medicines. It is logical to expect that non-psychological treatments designed for humans may also work on animals.

\section{Citation impact of research about alternative medicine}

In most broad Scopus fields where there is little research into the four alternative medicines, the few papers published attract less citations than average (Fig. 3). Nevertheless, the situation is more balanced for the fields in which it is more common. Other than 


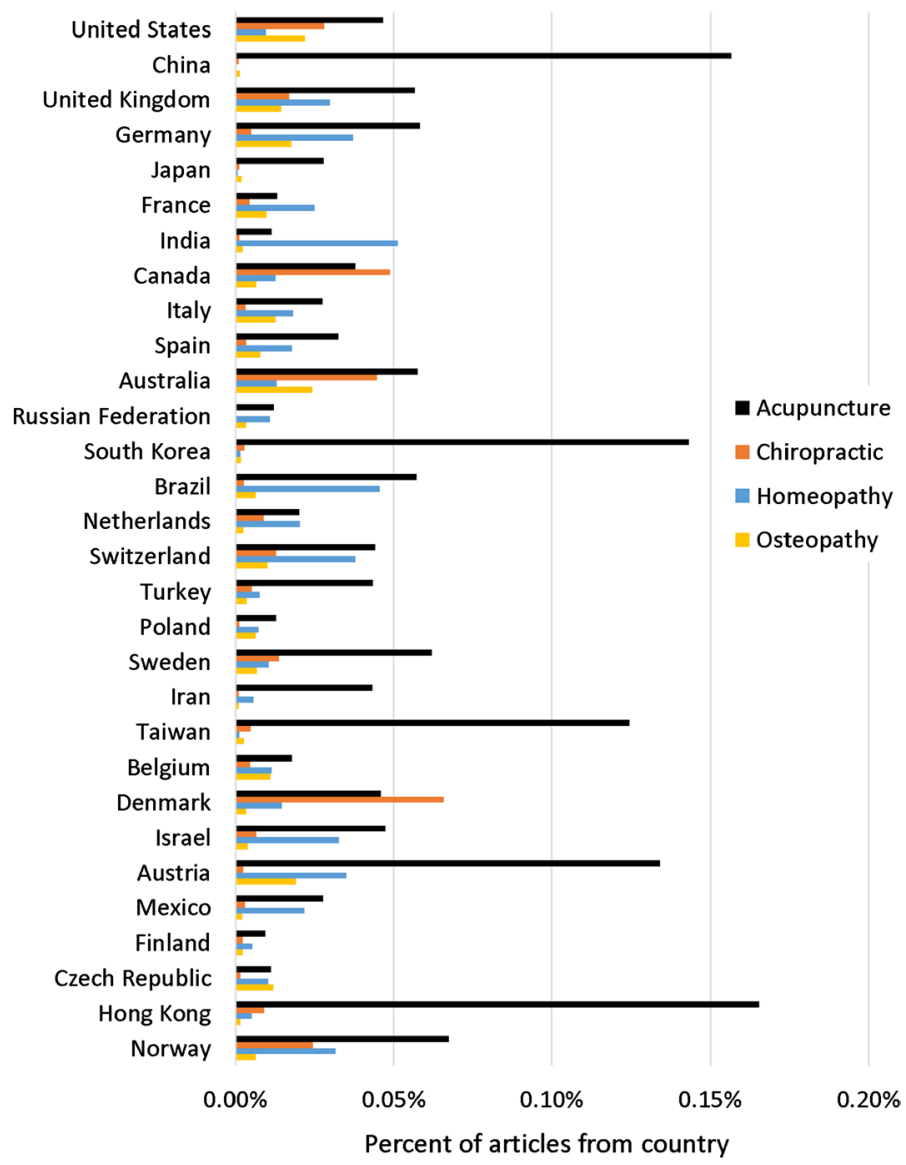

Fig. 1 Percentage of Scopus-indexed journal articles mentioning acupuncture, chiropractic, homeopathy or osteopathy 1996-2020 in the 30 countries with the most Scopus-indexed journal articles 1996-2020, based on all article authors

osteopathy, there is at least one broad field for which each alternative medicine tends to be cited above average. In particular, acupuncture and homeopathy seem to be more cited than average in Health Professions and Nursing. Chiropractic research tends to be more cited than average in Medicine, Veterinary, Neuroscience, Biochemistry, Genetics and Molecular Biology, and Social Sciences.

\section{Changes over time}

Graphs of changes in time in the prevalence and average citation impact of each topic are given for the four broad fields with the most articles. Average citation impacts are reported for all years, but the data are unreliable after 2017 due to short citation windows (insufficient time to accrue enough citations for reasonable estimates). A complete set of $27 \times 4=108$ graphs for each field and alternative medicine is available in the online supplement (https://doi.org/10.6084/m9.figshare.14547213). 


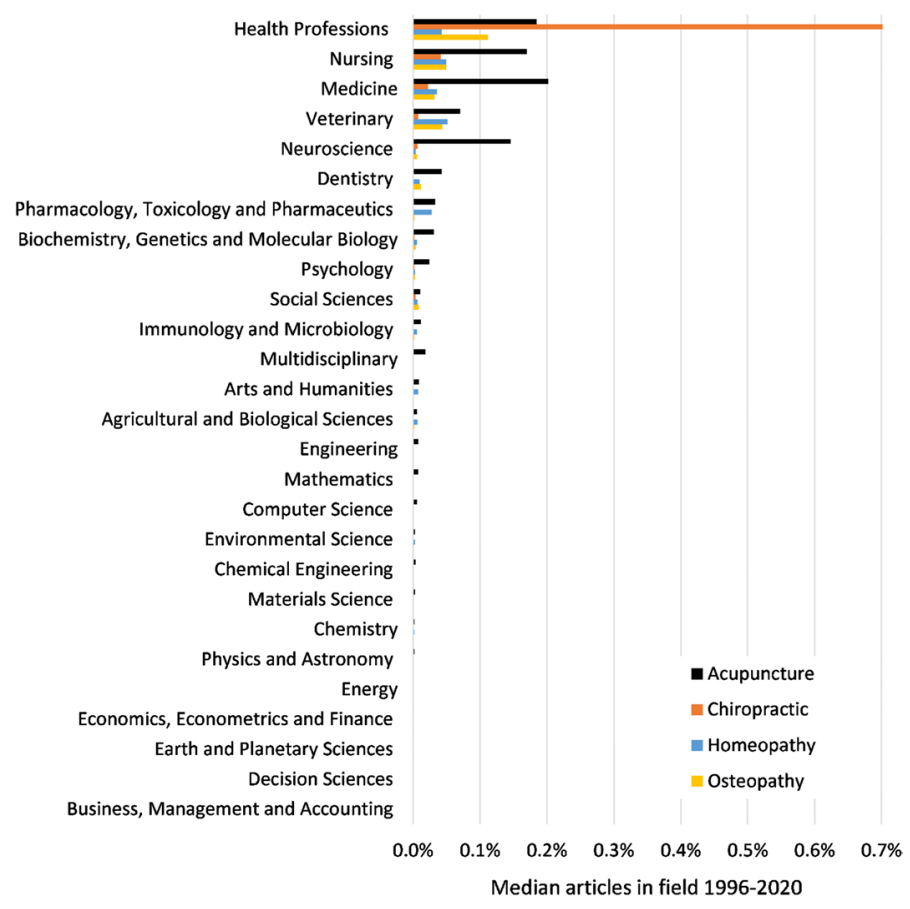

Fig. 2 Median (across years) percentage of Scopus-indexed journal articles mentioning acupuncture, chiropractic, homeopathy, or osteopathy 1996-2020 in each of 27 broad fields. Fields are ordered by decreasing sum of all four

\section{Acupuncture}

The prevalence and citation impact of acupuncture research within Scopus fields has varied over time, partly due to individual journal indexing or coverage changes (Fig. 4). The increase in the proportion of acupuncture research in 2005-2016 within Medicine was due to Scopus indexing the Zhongguo Zhen Jiu (Chinese Acupuncture \& Moxibustion) journal between these dates (Moxibustion is a traditional Chinese medicine often used with acupuncture). The indexing of this journal probably caused the decrease in average citation impact in 2005. In contrast, the decrease in prevalence of acupuncture within Nursing was mainly due to fewer articles being published in Explore: The Journal of Science and Healing, despite this journal continuing to be indexed (the overall number of articles per year decreased in this journal, not just for acupuncture). Also in contrast, the 2010 spike in Neuroscience was due to a Neurological Research issue supplement in 2010 entitled, "Advanced Acupuncture System for Neurological Disorders". Thus, due to the small numbers of articles published each year, the trends shown in the graphs may not reflect underlying levels of academic research interest.

\section{Chiropractic}

Chiropractic research seems to be decreasing in prevalence in its core fields (Fig. 5). Its citation impact has been consistently below average in the main field, Health Professions, 


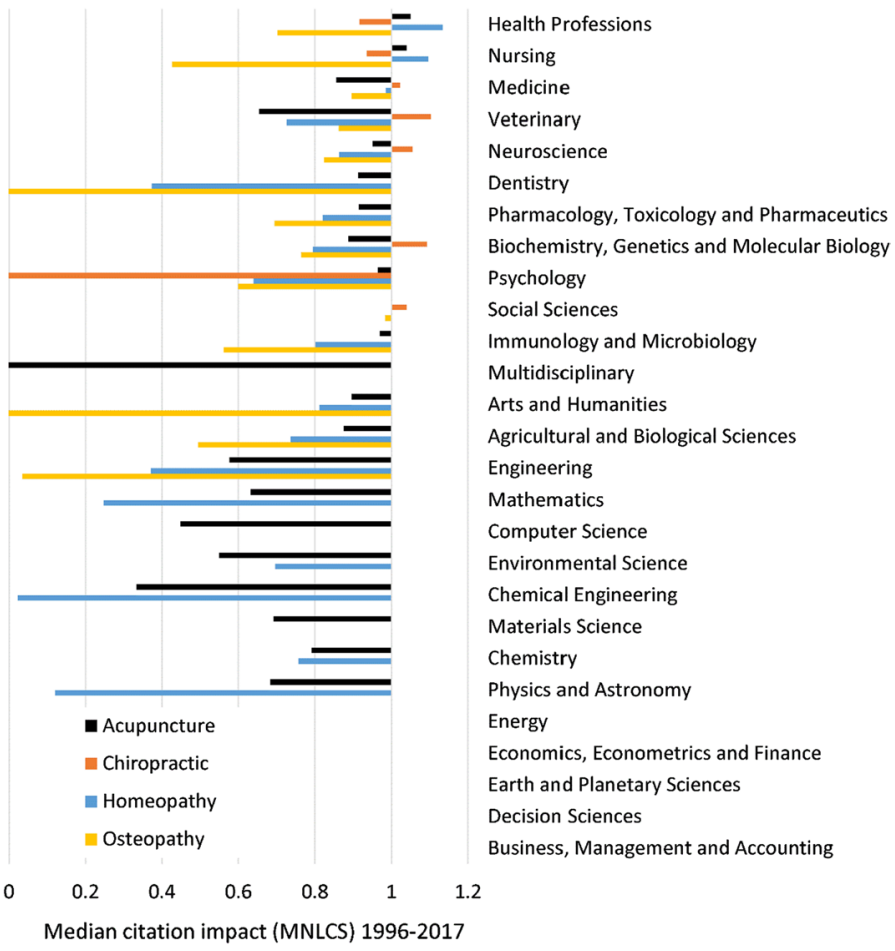

Fig. 3 Median (across years) average field and year normalised citation impact of Scopus-indexed journal articles mentioning acupuncture, chiropractic, homeopathy, or osteopathy 1996-2017 (1 is the average for all journal articles) in each of 27 broad fields. Fields are ordered as in Fig. 2

although the difference has not always been statistically significant. Within Medicine and Nursing, the citation impact of chiropractic research has not been consistently above or below average.

\section{Homeopathy}

Homeopathy seems to have decreased in prevalence in its two main fields, since 1999 in Health Professions and since 2005 in Nursing. Its prevalence may also be decreasing in Medicine. The decrease in popularity of acupuncture within Health Professions and Nursing was, as for acupuncture, partly due to fewer articles being published in Explore: The Journal of Science and Healing, despite this journal continuing to be indexed. In both cases the cause partly seems to be that homeopathy research has not increased at the same rate as the broad fields overall.

The marginally below average citation impact in Medicine was investigated further. One of the 46 narrow fields within the Scopus medicine category is Complementary and Alternative Medicine. Because the citation normalisation process is based on narrow fields for greater precision, a specialism can be cited above average because it tends to be more cited than other papers in its Scopus narrow field. Thus, homeopathy research could be cited above average for the Medicine broad field because it is more cited than the remainder of the Complementary and Alternative Medicine Scopus narrow field. The three most cited homeopathy papers 


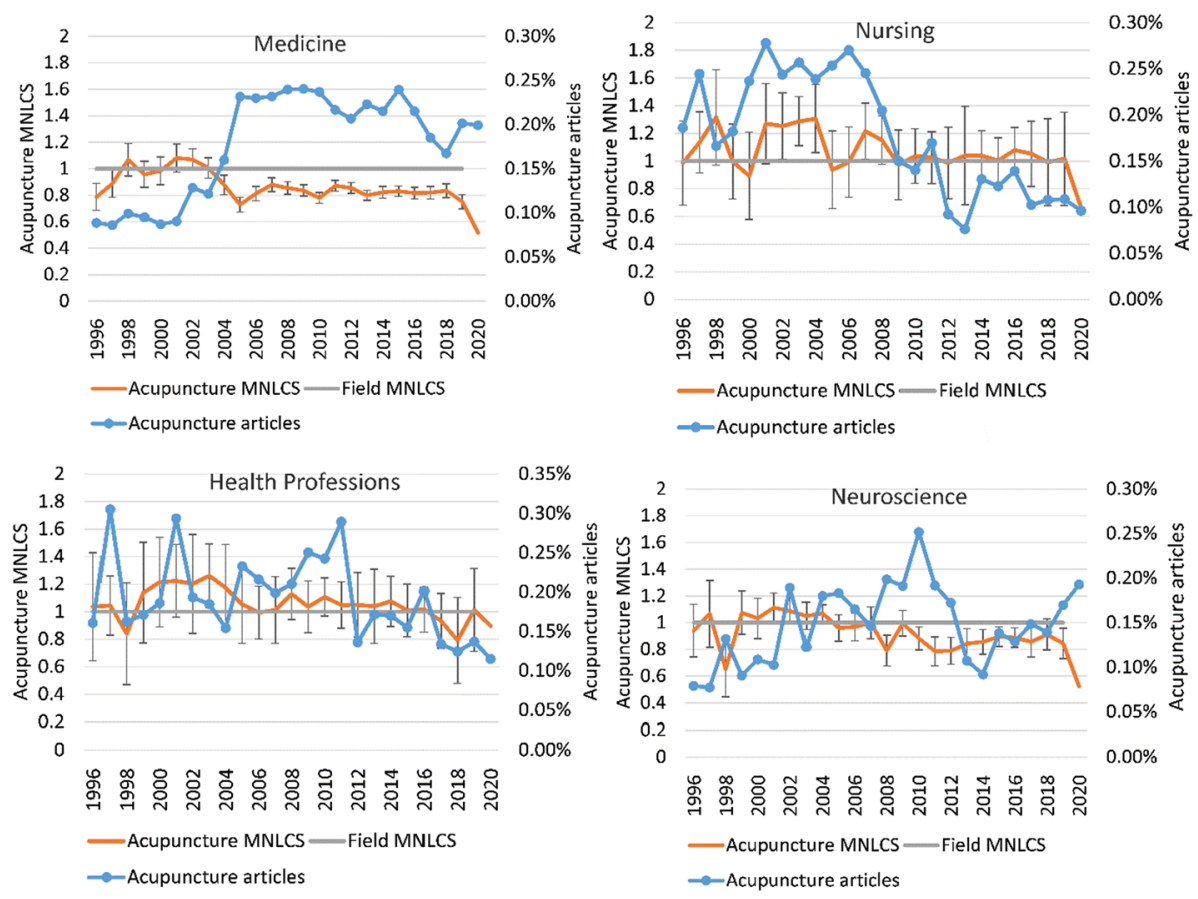

Fig. 4 Acupuncture: Medicine, Nursing, Health Professions, Neuroscience. Error bars indicate 95\% confidence intervals

in this category were all general surveys of an aspect of multiple alternative medicines (i.e., Ernst \& White, 2000; Robinson \& McGrail, 2004; Yamashita et al., 2002) rather than specific to homeopathy. Moreover, only three of the ten most cited papers mentioning homeopathy were specific to it. This suggests that the marginally below average citation average for homeopathy may not be supported by highly cited homeopathy research but may be due the scarcity of less cited research compared to other alternative medicines. Nevertheless, the fourth most cited paper was the discovery that six metal-based commercially produced homeopathic medicines at high dilutions contain small amounts of the starting materials (Chikramane et al., 2010). Another top cited paper confirmed that homeopathic remedies have few or no adverse effects (Dantas \& Rampes, 2000). Both have clear general relevance to homeopathy research.

The situation is similar for Health Professions and Nursing, which have above average citation impact homeopathy research. Only two of the ten most cited Health Professions papers are specific to homeopathy. There are two relevant narrow subfields of Health Professions in Scopus: Chiropractics and Complementary and Manual Therapy. Only two of the ten most cited Nursing papers are specific to homeopathy and there is no nursing Scopus narrow field about alterative medicines. Thus, the relatively high citation impact in these areas may not be primarily due to papers focusing on them being highly cited.

\section{Osteopathy}

Osteopathy seems to have increased in prevalence overall, especially in Health Professions and Nursing but also in Medicine (Fig. 7). This was due to an individual journal 

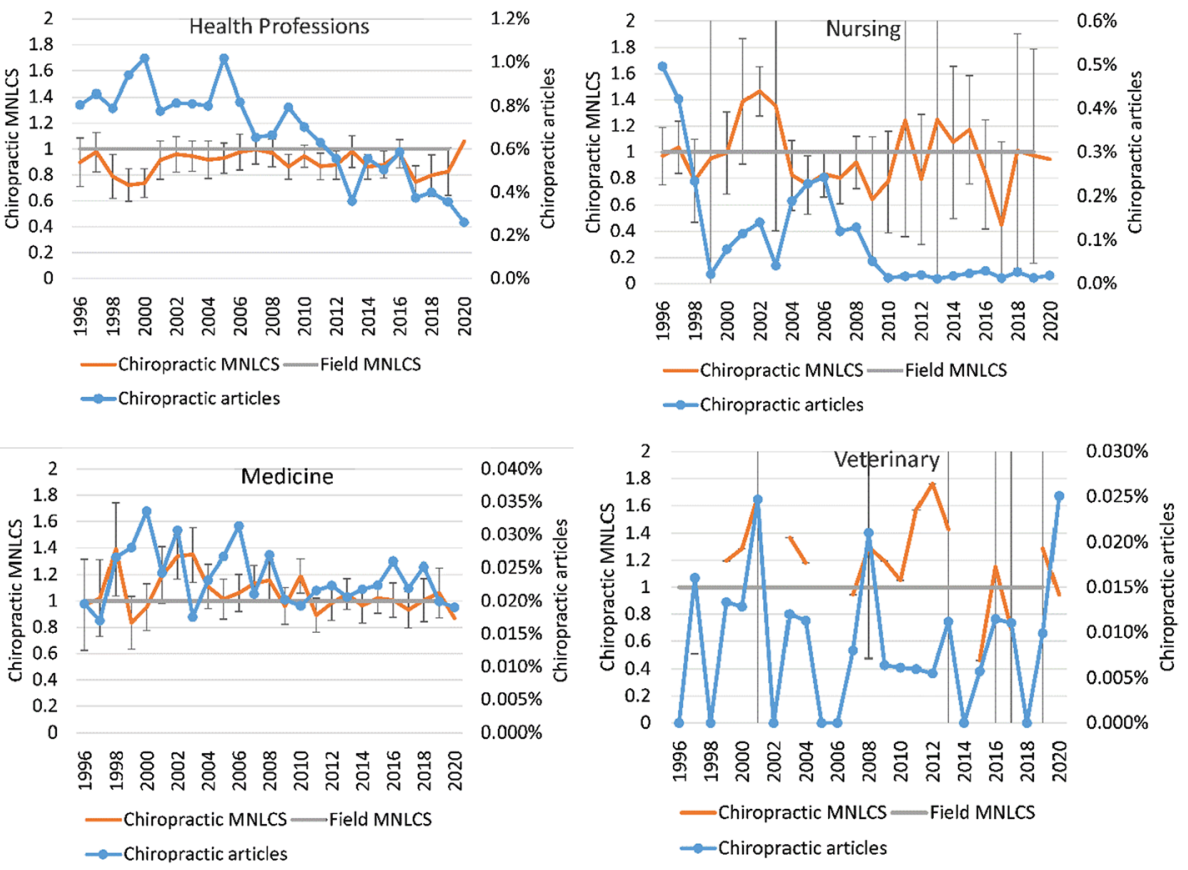

Fig. 5 Chiropractic: Health Professions, Nursing, Medicine, Veterinary. Error bars indicate 95\% confidence intervals

being added in the first two fields (American Academy of Osteopathy's AAO Journal indexed from 2006 to 2019 for both). The increase in prevalence of osteopathy research within Medicine coincided with a decline in average citation impact for most of the period reported. This might have occurred due to indexing new lower citation impact journals publishing some osteopathy.

\section{Discussion}

The results reveal huge international disparities in the extent to which the four alternative medicines are researched, with acupuncture being apparently the most common overall and dominant in East Asia (Fig. 1). Since interest in individual alternative therapies has varied greatly over time within countries, the results are not stable and may change in the future. The countries that seem to publish almost no research about the four alternative medicines may research other varieties, publish in national language journals outside Scopus, or tend to avoid alternative medicines altogether.

The international differences in prevalence of research are unsurprising given the (partially) known international differences in interest in them (WHO, 2019) and the bibliometric studies cited above (mainly for acupuncture) suggesting the dominance of Mainland China, the USA and, to a lesser extent, the UK and Germany (e.g., Ding \& Li, 2020; Şenel, 2019; Yang et al., 2019). The results here give finer-grained and updated information, including comparisons between four CAMs. The publication results may reflect 
international differences in the provision of higher education for practitioners or health professionals (e.g., nurses) when they are administered by organised health providers. Higher education provision might follow from efforts to standardise aspects of individual CAMs (e.g., Zollman \& Vickers, 1999). This last point is based on the assumption that higher education for a subject will tend to produce research into it as a by-product, at least partly because educators may need PhDs to gain employment or teach at Master's level.

The differences between fields in the amount of research mentioning each alternative medicine (Fig. 2) seem to reflect their health focus. The relatively high level of interest in acupuncture for neuroscience presumably derives from investigations of the effect of acupuncture needles on the brain, which would be instantaneous, in contrast to the other medicines. Some other areas with small amounts of relevant research are also plausible contributors, such as Engineering (of needles) for acupuncture. These small amounts reflect that most disciplines can in some way support many of the alternative medicines, even if they rarely do.

Given the low regard enjoyed by some or all alternative medicines from some scientists, it is unsurprising that research mentioning the four varieties investigated is less cited than average in most fields (Fig. 3). This partly conflicts with the previous citation evidence that CAM research does not tend to be published in low impact journals (Danell et al., 2020). Conversely, it may be surprising than any "pseudoscience" (as some think that some of these are) would be cited more than average, especially in the key broad field of Medicine. It is possible that CAM research published outside of its core areas would be more strongly disparaged because there are few alternative medicine specialists to cite it and others may consider that researching or citing the topic, or some areas of it, may cause them reputational damage. The literature review above shows that CAM papers are sometimes extensively published in non-CAM journals (e.g., Sleep, Cephalalgia). The above average citation rate for acupuncture and homeopathy in Nursing and Health Professions is hard to explain, although the citations presumably originate from acupuncture and homeopathy specialists that may have a culture of citing extensively within their field. The above average citation rate of chiropractic in Medicine and four other fields is also difficult to explain. Whilst acupuncture and chiropractic have some scientific evidence of value for some applications, these alternative medicines and homeopathy have a wide variety of claimed benefits, which should, in theory reduce their overall citation impact. For alternative medicine papers in non-specialist journals, it is possible that the research needs to be higher quality or wider in scope to be published because of the stigma that might otherwise attach to reviewers or referees recommending accepting it. For example, whilst a pilot study of a scientifically motivated treatment might be accepted in a general journal, homeopathy research might need a randomised controlled trial to demonstrate publishable scientific credentials.

It is difficult to draw strong conclusions about trends in prevalence or impact of the four types of alternative medicine research (Figs. 4, 5, 6 and 7) because there are few papers published per year, particularly within broad fields. Moreover, these papers are often concentrated in specialist journals and the inclusion or exclusion of these journals from Scopus can cause step changes in their apparent prevalence and citation impact. Within Medicine and Health Professions, the inclusion of CAM narrow field categories means that CAM research is partly norm referenced against itself for citations, weakening any disadvantage that the research might have from the perspective of citation analysis. Adding to this the fact that key highly cited papers about individual alternative medicines sometimes cover multiple types or discuss alternative medicine in general, it is difficult to draw strong conclusions from field and year normalised citation counts for any specialty. This 
is a generic limitation for any research area that is large and distinctive enough to have its own category.

\section{Conclusions}

The results confirm that there are large international differences in the amount of research into the four different CAM specialities. These are presumably associated with their national prevalence and the provision of higher education training for practitioners. The results also suggest that research into acupuncture, chiropractic, homeopathy, and osteopathy tends to generate below average scientific impact, in the form of citations, except in the core Health Professions and Nursing areas for acupuncture and homeopathy, and in Medicine, Veterinary, Neuroscience, Biochemistry, Genetics and Molecular Biology, and Social Sciences for chiropractic. The results are complicated by changes in the journals indexed by Scopus and the existence of alternative medicine narrow field categories in Scopus, but the results overall show that alternative medicine research can sometimes generate adequate citation impact in its core areas.

In terms of practical conclusions, the results suggest that for institutional scholarly reputation or academic careers, alternative medicine research is not a substantial disadvantage from a citation analysis perspective for researchers within its core areas, although it might be from other perspectives, and for other CAM varieties. The results also point to the importance of not interpreting citation counts as strong indicators of academic impact or
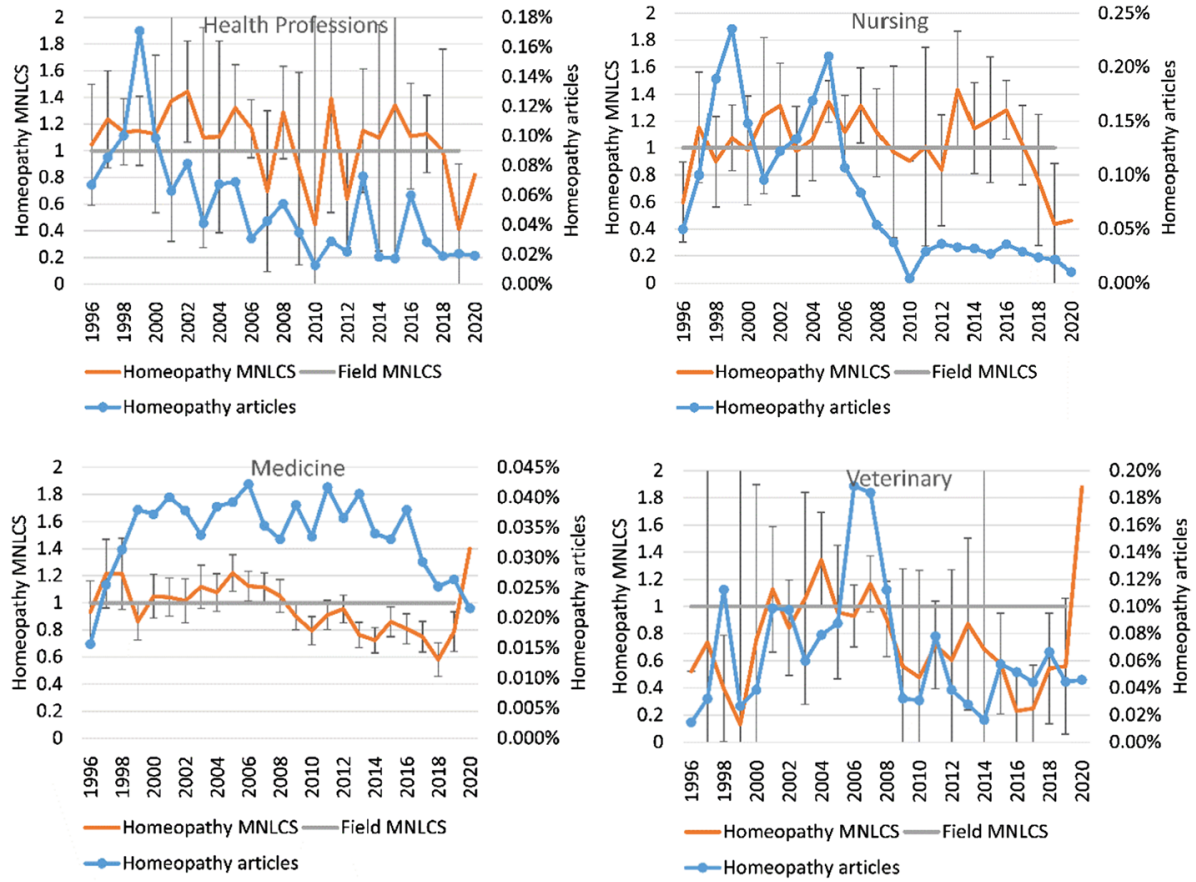

Fig. 6 Homeopathy: Health Professions, Nursing, Medicine, Veterinary. Error bars indicate 95\% confidence intervals 

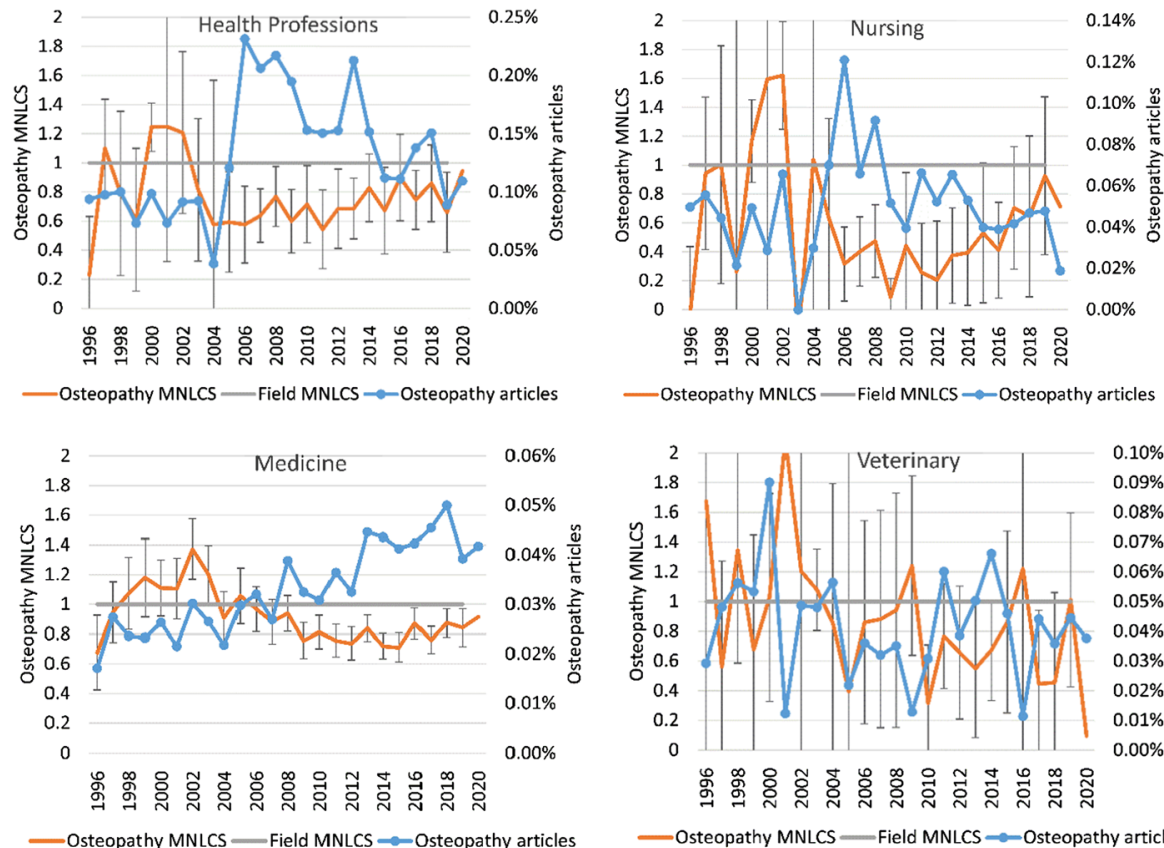

Osteopathy MNLCS —Field MNLCS $\multimap$ Osteopathy articles

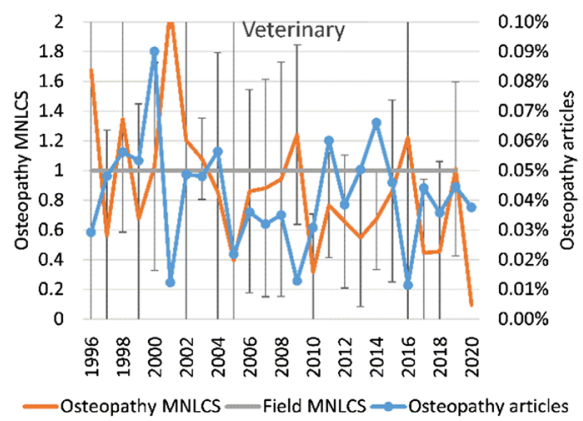

Fig. 7 Osteopathy: Health Professions, Nursing, Medicine, Veterinary. Error bars indicate 95\% confidence intervals

value because of the factors that can influence their value. In addition, if it is believed that research about at least one of acupuncture, chiropractic, or homeopathy has little value, then it is concerning that it has above average citation impact in at least one broad field. Thus, evaluating the citation impact of a specialty will not necessarily help to determine its value.

\section{References}

Anonymous. (1979). The essentials of Chinese acupuncture. Foreign Languages Press.

BBC (2018). NHS-funded homeopathic treatment to end in Bristol. https://www.bbc.co.uk/news/uk-engla nd-bristol-45028622

Bougioukas, K. I., Vounzoulaki, E., Mantsiou, C. D., Papanastasiou, G. D., Savvides, E. D., Ntzani, E. E., \& Haidich, A. B. (2021). Global mapping of overviews of systematic reviews in healthcare published between 2000 and 2020: A bibliometric analysis. Journal of Clinical Epidemiology, 137(1), 58-72.

Chikramane, P. S., Suresh, A. K., Bellare, J. R., \& Kane, S. G. (2010). Extreme homeopathic dilutions retain starting materials: A nanoparticulate perspective. Homeopathy, 99(4), 231-242.

Colquhoun, D., \& Novella, S. P. (2013). Acupuncture is theatrical placebo. Anesthesia \& Analgesia, 116(6), 1360-1363.

Danell, J. A. B., Danell, R., \& Vuolanto, P. (2020). Fifty years of complementary and alternative medicine (CAM)-a bibliometric analysis of publication activity and general content of the publications. Journal of Scientometric Research, 9(3), 268-276.

Dantas, F., \& Rampes, H. (2000). Do homeopathic medicines provoke adverse effects? A systematic review. British Homoeopathic Journal, 89, S35-S38.

Davis, N. \& Campbell, D. (2017). 'A misuse of scarce funds': NHS to end prescription of homeopathic remedies. Guardian. https://www.theguardian.com/lifeandstyle/2017/jul/21/a-misuse-of-scarce-fundsnhs-to-end-prescription-of-homeopathic-remedies 
Ding, Z., \& Li, F. (2020). Publications in integrative and complementary medicine: A ten-year bibliometric survey in the field of ICM. Evidence-Based Complementary and Alternative Medicine, 2020, 4821950. https://doi.org/10.1155/2020/4821950

Ernst, E. (2000). Prevalence of use of complementary/alternative medicine: A systematic review. Bulletin of the World Health Organization, 78, 258-266.

Ernst, E. (2008). Chiropractic: A critical evaluation. Journal of Pain and Symptom Management, 35(5), $544-562$.

Ernst, E., \& White, A. (2000). The BBC survey of complementary medicine use in the UK. Complementary Therapies in Medicine, 8(1), 32-36.

Falkenberg, T., Lewith, G., di Sarsina, P. R., von Ammon, K., Santos-Rey, K., Hök, J., \& Uehleke, B. (2012). Towards a pan-European definition of complementary and alternative medicine-a realistic ambition? Complementary Medicine Research, 19(Suppl. 2), 6-8. https://doi.org/10.1159/000343812

Franke, H., Franke, J. D., \& Fryer, G. (2014). Osteopathic manipulative treatment for nonspecific low back pain: A systematic review and meta-analysis. BMC Musculoskeletal Disorders. https://doi.org/10. 1186/1471-2474-15-286

Fu, J. Y., Zhang, X., Zhao, Y. H., Huang, M. H., \& Chen, D. Z. (2011). Bibliometric analysis of complementary and alternative medicine research over three decades. Scientometrics, 88(2), 617-626.

Gao, Z., Zhang, J., Liu, G. F., \& Ji, L. X. (2021). Research trends from 2010 to 2020 for pain treatment with acupuncture: A bibliometric analysis. Journal of Pain Research, 14, 941-952. https://doi.org/10.2147/ JPR.S300911

Kassab, S., Cummings, M., Berkovitz, S., van Haselen, R., \& Fisher, P. (2009). Homeopathic medicines for adverse effects of cancer treatments. Cochrane Database of Systematic Reviews. https://doi.org/10. 1002/14651858.CD004845.pub2

Klein, S. D., Würtenberger, S., Wolf, U., Baumgartner, S., \& Tournier, A. (2018). Physicochemical investigations of homeopathic preparations: A systematic review and bibliometric analysis-Part 1. The Journal of Alternative and Complementary Medicine, 24(5), 409-421.

Larivière, V., Desrochers, N., Macaluso, B., Mongeon, P., Paul-Hus, A., \& Sugimoto, C. R. (2016). Contributorship and division of labor in knowledge production. Social Studies of Science, 46(3), 417-435.

Li, R., Sun, J., Hu, H., Zhang, Q., Sun, R., Zhou, S., \& Fang, J. (2020). Research trends of acupuncture therapy on knee osteoarthritis from 2010 to 2019: A bibliometric analysis. Journal of Pain Research, 13, 1901-1913. https://doi.org/10.2147/JPR.S258739

Liang, Y. D., Li, Y., Zhao, J., Wang, X. Y., Zhu, H. Z., \& Chen, X. H. (2017). Study of acupuncture for low back pain in recent 20 years: A bibliometric analysis via CiteSpace. Journal of Pain Research, 10, 951-964. https://doi.org/10.2147/JPR.S132808

Linde, K., Allais, G., Brinkhaus, B., Fei, Y., Mehring, M., Shin, B.-C., Vickers, A., \& White, A. R. (2016). Acupuncture for the prevention of tension-type headache. Cochrane Database of Systematic Reviews. https://doi.org/10.1002/14651858.CD007587.pub2

Linde, K., Allais, G., Brinkhaus, B., Fei, Y., Mehring, M., Vertosick, E. A., Vickers, A., \& White, A. R. (2016). Acupuncture for the prevention of episodic migraine. Cochrane Database of Systematic Reviews. https://doi.org/10.1002/14651858.CD001218.pub3

Long, W., Li, Y., Pei, Yu., Li, H., Ma, S., Liu, S., Liu, M., \& Wen, Y. (2021). The application of acupuncture in obstetrics and gynecology: A bibliometric analysis based on web of science. Annals of Palliative Medicine, 10(3), 3194-3204.

Martín-Martín, A., Thelwall, M., Orduna-Malea, E., \& López-Cózar, E. D. (2021). Google scholar, microsoft academic, scopus, dimensions, web of science, and opencitations' COCI: A multidisciplinary comparison of coverage via citations. Scientometrics, 126(1), 871-906.

Mathie, R. T., Frye, J., \& Fisher, P. (2015). Homeopathic Oscillococcinum ${ }^{\circledR}$ for preventing and treating influenza and influenza-like illness. Cochrane Database of Systematic Reviews. https://doi.org/10. 1002/14651858.CD001957.pub6

Merton, R. K. (1973). The sociology of science: Theoretical and empirical investigations. University of Chicago press.

Moed, H. F. (2006). Citation analysis in research evaluation. Springer Science \& Business Media.

Mongeon, P., \& Paul-Hus, A. (2016). The journal coverage of web of science and scopus: A comparative analysis. Scientometrics, 106(1), 213-228.

Mongeon, P., Smith, E., Joyal, B., \& Larivière, V. (2017). The rise of the middle author: Investigating collaboration and division of labor in biomedical research using partial alphabetical authorship. PloS One, 12(9), e0184601.

NHMRC (2015). Evidence on the effectiveness of homeopathy for treating health conditions. Canberra: NHMRC https://nhmrc.gov.au/about-us/publications/homeopathy

NHS (2021). Acupuncture. https://www.nhs.uk/conditions/acupuncture/ 
Pei, W., Peng, R., Gu, Y., Zhou, X., \& Ruan, J. (2019). Research trends of acupuncture therapy on insomnia in two decades (from 1999 to 2018): A bibliometric analysis. BMC Complementary and Alternative Medicine, 19(1), 1-9.

Pohlman, K. A., Potocki, E. N., \& Lawrence, D. J. (2009). A descriptive analysis and checklist critique of the articles in the journal of clinical chiropractic pediatrics, 1996 to 2007. Journal of Manipulative and Physiological Therapeutics, 32(8), 654-659.

Relton, C., Cooper, K., Viksveen, P., Fibert, P., \& Thomas, K. (2017). Prevalence of homeopathy use by the general population worldwide: A systematic review. Homeopathy, 106(2), 69-78.

Robinson, A., \& McGrail, M. R. (2004). Disclosure of CAM use to medical practitioners: A review of qualitative and quantitative studies. Complementary Therapies in Medicine, 12(2-3), 90-98.

Scopus (2021). How scopus works. https://www.elsevier.com/solutions/scopus/how-scopus-works/content

Şenel, E. (2019). Evolution of homeopathy: A scientometric analysis of global homeopathy literature between 1975 and 2017. Complementary Therapies in Clinical Practice, 34, 165-173.

Silvello, G. (2018). Theory and practice of data citation. Journal of the Association for Information Science and Technology, 69(1), 6-20.

Steel, A., Sundberg, Tobias, Reid, R., Ward, L., Bishop, F. L., Leach, M., Cramer, H., Wardle, J., \& Adams, J. (2017). Osteopathic manipulative treatment: A systematic review and critical appraisal of comparative effectiveness and health economics research. Musculoskeletal Science and Practice, 27, 165-175. https://doi.org/10.1016/j.math.2016.10.067

Stochkendahl, M. J., Rezai, M., Torres, P., Sutton, D., Tuchin, P., Brown, R., \& Côté, P. (2019). The chiropractic workforce: A global review. Chiropractic \& Manual Therapies, 27(1), 1-9.

Thelwall, M. (2017). Three practical field normalised alternative indicator formulae for research evaluation. Journal of Informetrics, 11(1), 128-151.

Thelwall, M. (2018). Dimensions: A competitor to scopus and the web of science? Journal of Informetrics, $12(2), 430-435$.

Treister-Goltzman, Y., \& Peleg, R. (2015). Trends in publications on complementary and alternative medicine in the medical literature. Journal of Complementary and Integrative Medicine, 12(2), 111-115.

Vera-Baceta, M. A., Thelwall, M., \& Kousha, K. (2019). web of science and scopus language coverage. Scientometrics, 121(3), 1803-1813.

Walker, B. F., French, S. D., Grant, W., \& Green, S. (2010). Combined chiropractic interventions for lowback pain. Cochrane Database of Systematic Reviews. https://doi.org/10.1002/14651858.CD005427. pub2

Wang, J. (2013). Citation time window choice for research impact evaluation. Scientometrics, 94(3), $851-872$.

White, A. (2009). Western medical acupuncture: A definition. Acupuncture in Medicine, 27(1), 33-35.

WHO. (2019). WHO Global report on traditional and complementary medicine 2019. WHO.

Yamashita, H., Tsukayama, H., \& Sugishita, C. (2002). Popularity of complementary and alternative medicine in Japan: A telephone survey. Complementary Therapies in Medicine, 10(2), 84-93.

Yang, W., Hao, X., Qu, J., Wang, L., Zhang, M., Jiang, Y., \& Liu, Y. (2019). Collaborative networks and thematic trends of research on the application of complementary and alternative medicine in cancer patients: A bibliometric analysis. Complementary Therapies in Clinical Practice, 37, 58-67.

Zhang, J., Zhang, Y., Hu, L., Huang, X., Liu, Y., Li, J., \& Yu, H. (2020). Global trends and performances of magnetic resonance imaging studies on acupuncture: A bibliometric analysis. Frontiers in Neuroscience, 14, 620555. https://doi.org/10.3389/fnins.2020.620555

Zhao, T., Guo, J., Song, Y., Chen, H., Sun, M., Chen, L., \& Sun, J. (2021). A bibliometric analysis of research trends of acupuncture therapy in the treatment of migraine from 2000 to 2020. Journal of Pain Research, 14, 1399-1414. https://doi.org/10.2147/JPR.S306594

Zollman, C., \& Vickers, A. (1999). What is complementary medicine? BMJ, 319(7211), 693-696. 